Review

\title{
Decrease of Electrical Systole of Heart: A Review of more than 300 Patients
}

\author{
Francisco R. Breijo-Márquez
}

How to cite this paper: Breijo-

Márquez, F. R. (2021). Decrease of Electrical Systole of Heart: A Review of more than 300 Patients. Global Journal of Cardiovascular Diseases, 1(1), 1-9. Retrieved from

https://www.scipublications.com/jou $\mathrm{rnal} /$ index.php/gjcd/article/view/118

Received: July 22, 2021

Accepted: August 31, 2021

Published: September 1, 2021

Copyright: (c) 2021 by the authors. Submitted for possible open access publication under the terms and conditions of the Creative Commons Attribution (CC BY) license (http://creativecommons.org/licenses/by/4.0/).
East Boston Hospital, School of Medicine, 02136, Tremont ST. Boston Massachusetts, USA

*Correspondence: frbreijo@gmail.com

\begin{abstract}
Alterations in the cardiac electrical system are the cause of morbidity and a wide variety of symptoms - from mild to those requiring urgent intervention - because of the risk of sudden cardiac death. The most prevalent of these types of electrical disturbances is atrial fibrillation, the incidence of which is growing as the average age of the human being increases. Among many other electrical heart alterations is one I describe in this document: "The presence of short PR and QTc intervals together in the same ECG tracing". Methods: A cardiac calibrator was used by four different cardiologists in blinded fashion to document a distinct ECG pattern, that of a short PR and QTc intervals together in the same ECG tracing from more than 2.500 cases assessed for this condition (more than two thousand five hundred cases evaluated since 2.007 to date). Results: Here we describe the clinical features of 330 patients with a documented short PR and QTc intervals together in the same ECG tracing along with descriptions of their symptoms and ancillary investigations. Conclusions: ECG tracing must be studied carefully in patients with suggestive symptoms before declaring normal an ECG tracing with certain defined characteristics.
\end{abstract}

Keywords: PR and QT intervals; Syncope; Nocturnal palpitations; Cardiac arrest; Misdiagnosis

\section{Objective}

By means of this document, the author and his full research team would like to point out to health care professionals -both cardiologists and non-cardiologists- that the electrocardiographic entity of a simultaneous short PR interval and short QT interval is far than an anecdote, but instead it is a common and important condition, with, in our files, more than two thousand individuals with the electrocardiographic of a short PQ interval and a short QTc interval in the same electrocardiographic tracing, that is, a "decrease of the cardiac electrical systole". In this report, more than three hundred individuals fulfilling these ECG requirements are presented, along with their most frequent clinical features.

\section{Introduction}

In 2002, Dr. Mazhari et al. [1] described the gene responsible for an electrocardiographic anomaly, the KCNE3 gene, in its ectopic expression. Both the short PR interval and the short QT interval are being extensively studied and sought in routine practice on a daily basis - but always as isolated entities, not together in the same ECG tracing [2]. Regarding the short PR interval [3], Wolf- Parkinson-White [4], Lown-GanongLevine [5], Mannheim [6] and others, have reported extremely important cardiac electrical entities.

\section{Methods}

Electrocardiographic tracings were measured by a single cardiac calibrator and evaluated by independently by four cardiology experts - including the author- in a 
"blinded" way. Only those tracings that showed a PR interval length of fewer than 120 milliseconds and a QTc interval length (Bazzett, Fridericia, Hodges, Framingham) of less than 360 milliseconds in males and less than 370 in females according to Gollop et al. [3] were included.

\section{Results}

330 patients from our practice were identified as carrying the described ECG pattern, Describing each case with its images is impossible, therefore, we will illustrate with the most significant cases. The first image (Figure 1 and Figure 2) belongs to the first ECG tracing that we detected about the year 2007. It belonged to a 37 year-old male who reported many of the above-mentioned symptoms, until ultimately he had an unrecoverable cardiac arrest and sudden cardiac death.

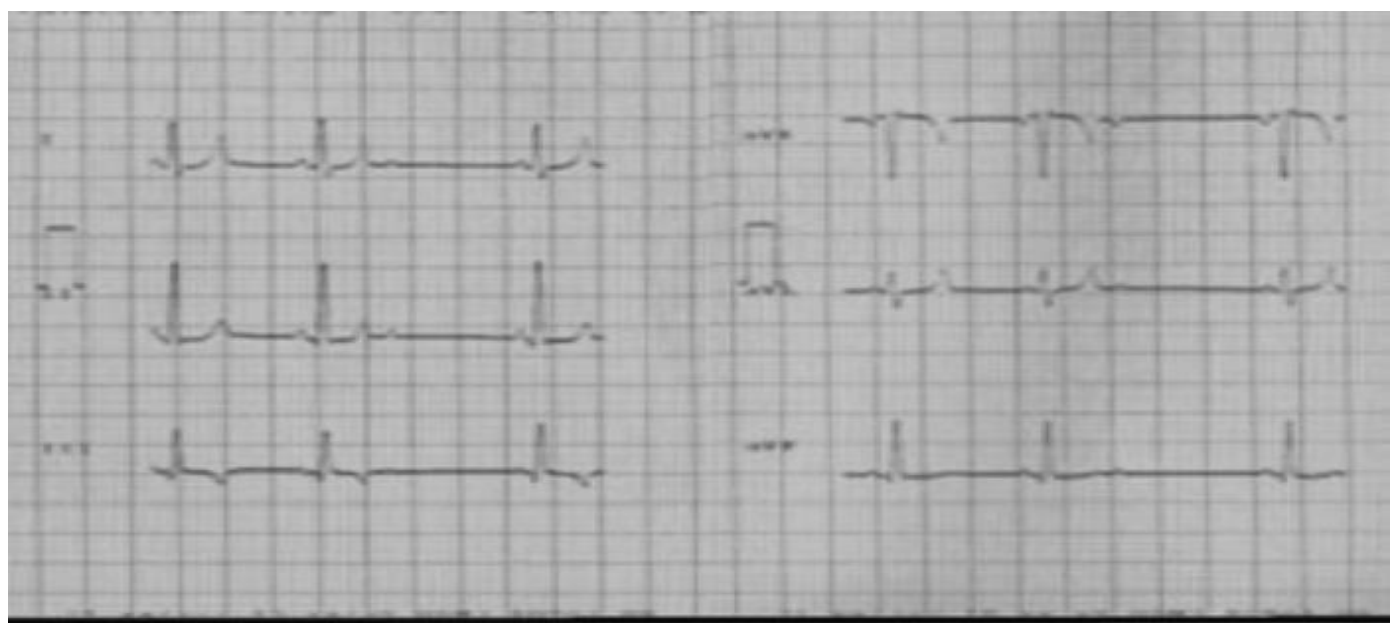

Figure 1. [8]: The short PR and QT intervals in the context of a second degree A-V block (in member leads).

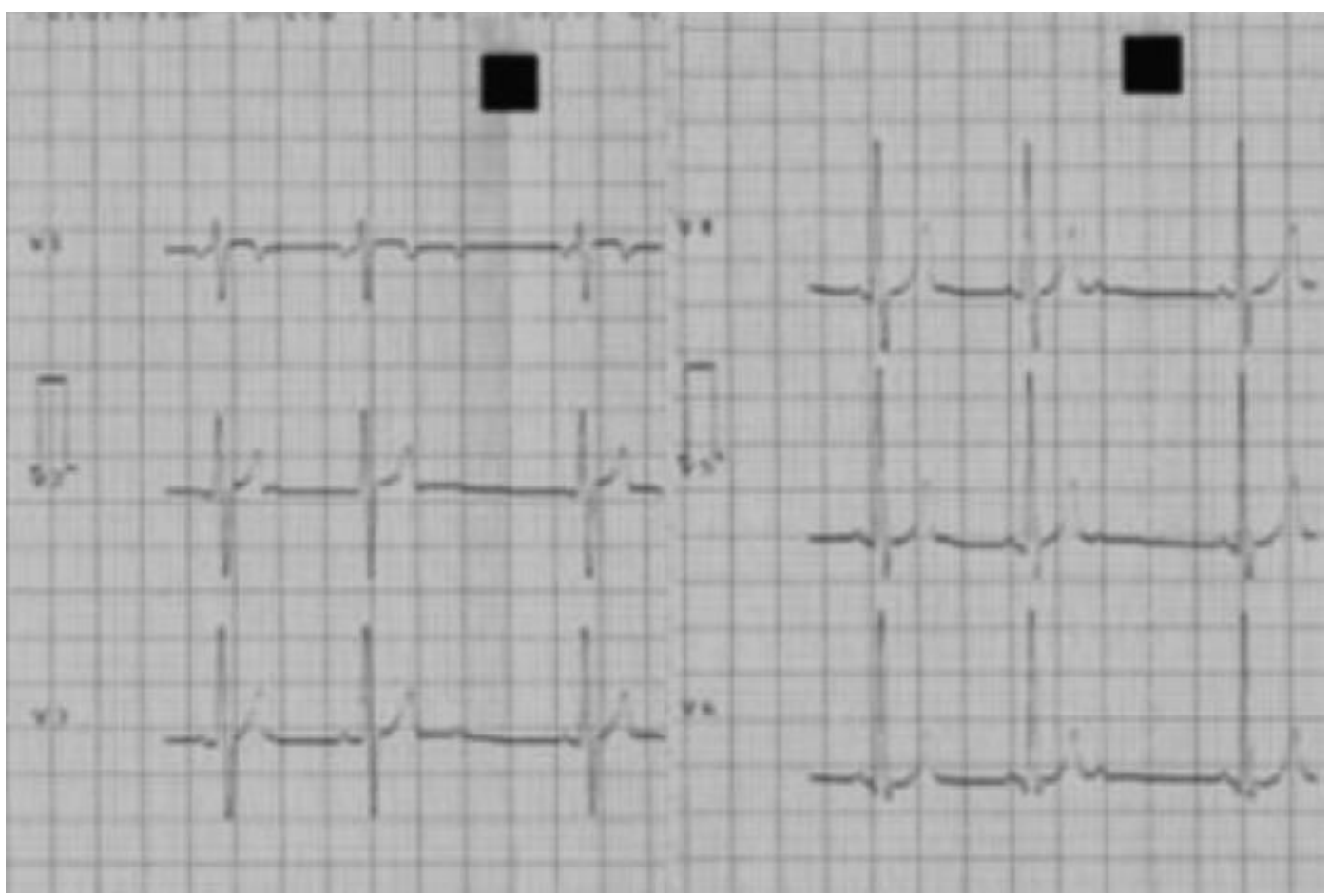

Figure 2. Identical electrocardiographic tracing as Figure 1, but in the precordial leads. 


\subsection{Results of the measurement with various cardiac calibrators}

* Average values obtained from PR interval: 100-110 milliseconds.

* Average values obtained from QT corrected with several formulas:

\begin{tabular}{l} 
RR 0.594059405940594 \\
QTC (Rautaharju) 326 \\
QTc (Bazett) 315 \\
QTC (Framingham) 243 \\
QTc (Friderica) 289 \\
QTC (Call) 299 \\
\hline
\end{tabular}

The observation of a short PR interval and a short QT interval on the same electrocardiographic tracing (that is, on the same person) is evident.

In some of the 330 electrocardiographic tracings that we considered as fulfilling our criteria of a short PR interval together with a short QTc interval in the same individual; the pattern occurred together with other cardiological entities, including W.P.W [8], a Wellens pattern [9] and other entities. In one case, whose image is shown, a more than possible Mobitz Type II is seen.

All patients underwent further investigation, such as Holter (Figure 4), tilt test, echocardiography, and in some even an electrogram (EEF below) (Figure 3).

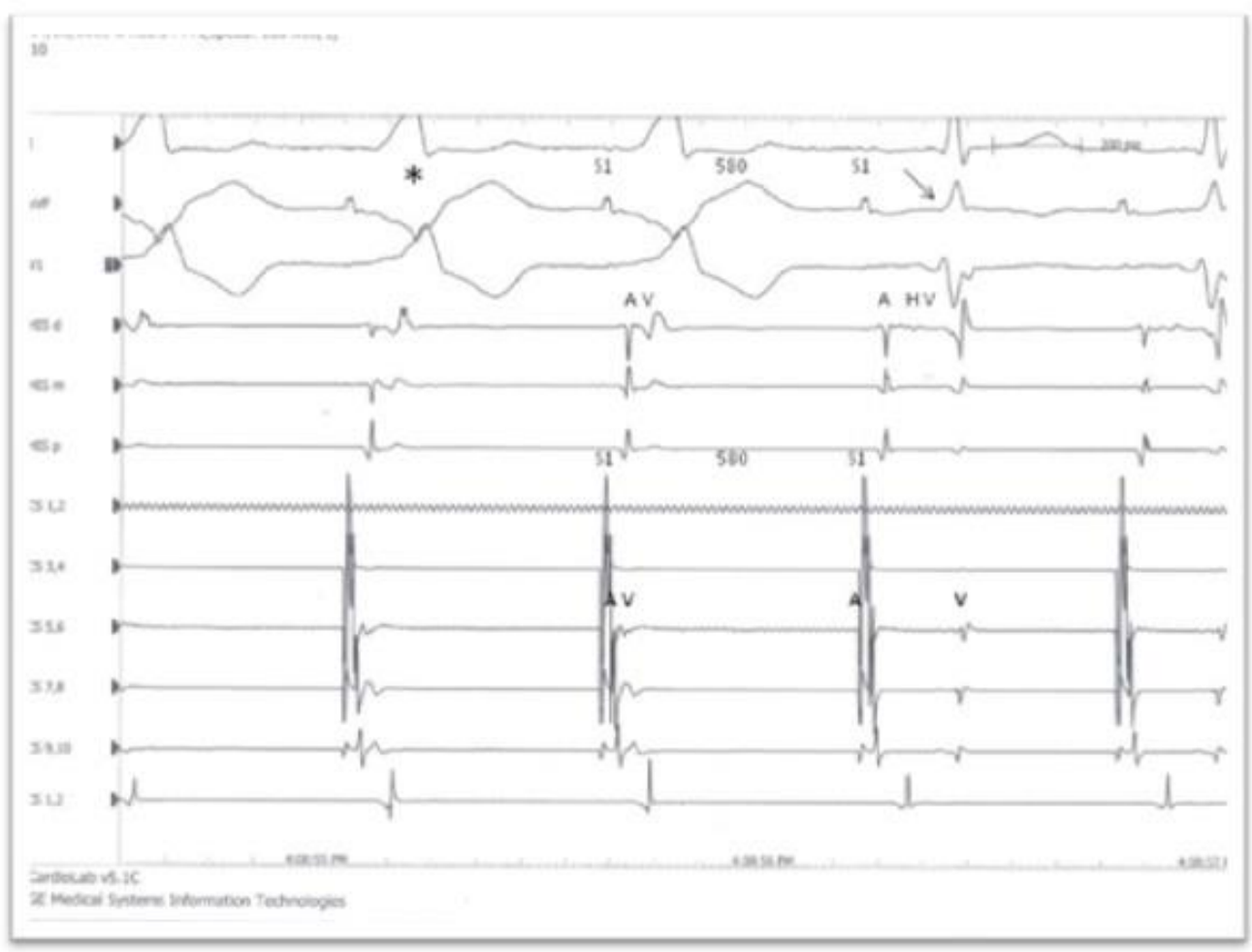

Figure 3. Electrophysiological study in detail. 


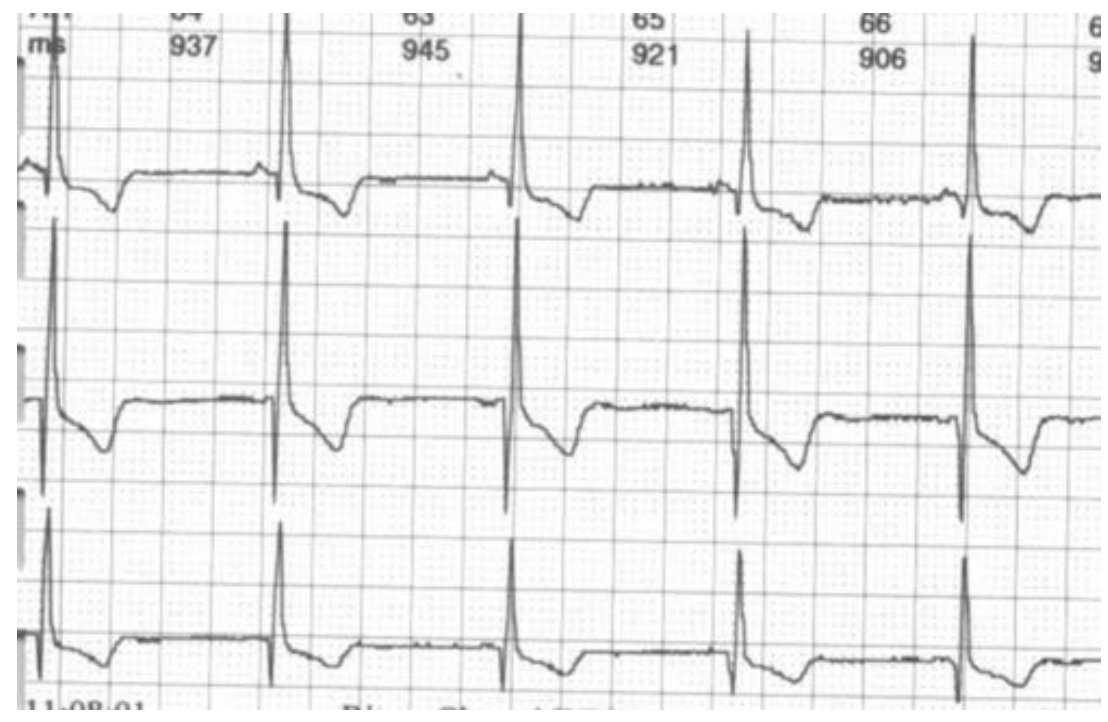

Figure 4. Detail of a Holter study

The presence of a short PR interval together with a short QT interval is evident and quantifiable.

The second image we illustrate below was the second case we diagnosed: It is striking that at first, the visualization of the ECG tracing seems to be normal, but this is not the case; he was a carrier of this entity, with different types of ill-defined symptoms, predominantly nocturnal palpitations.

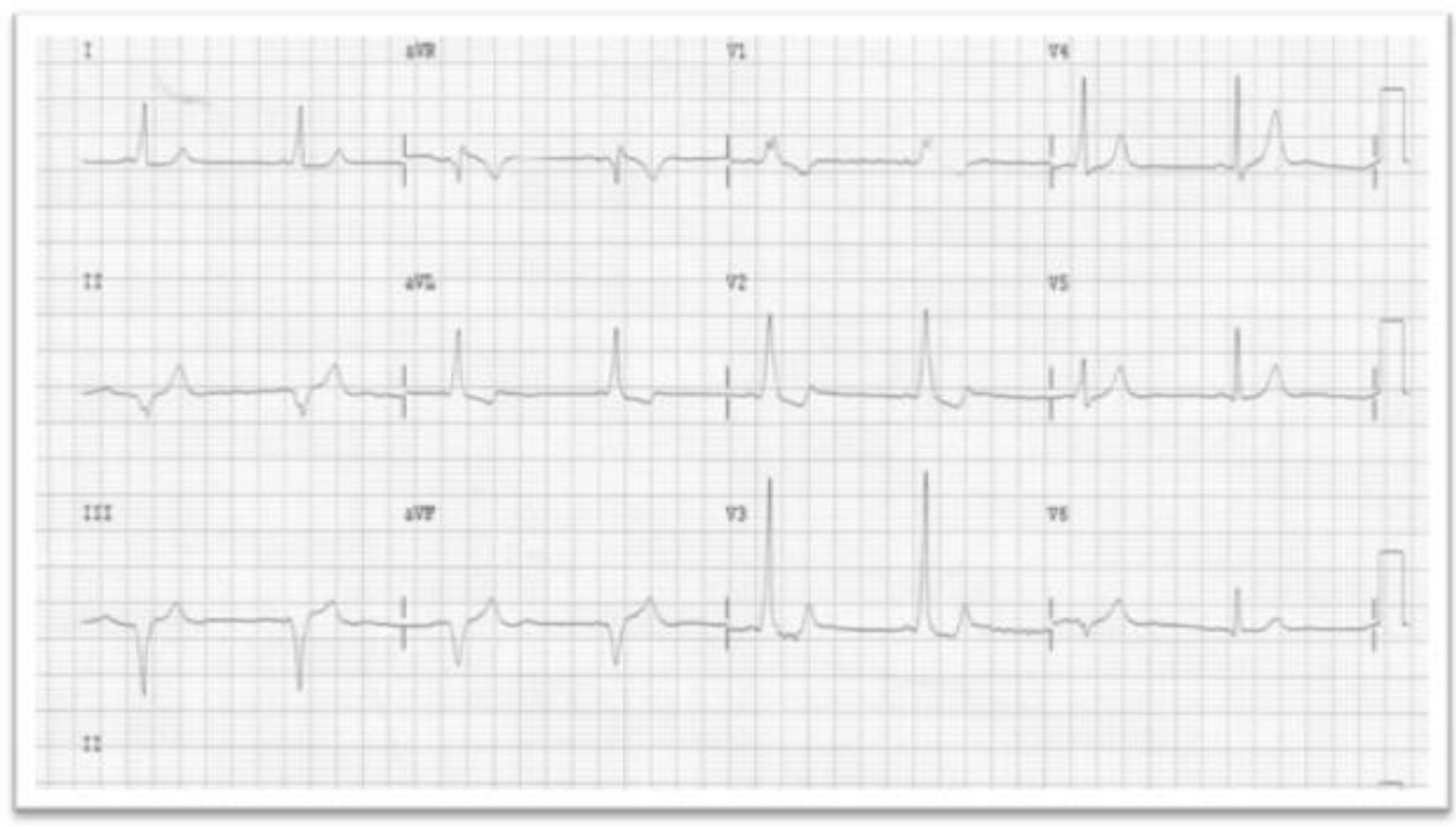

Figure 5. A complete ECG tracing.

From a patient who is, currently, baseline conditions, and nevertheless he had many episodes of tachyarrhythmias, mainly ventricular tachycardias that were optimally recovered. The graphic image represented here was taken 18 hours after the cardiac arrest. Values PR interval: 100-110 msec. 
Values QTc:

RR 1.2
QTC (Rautahariu) 437
QTC (Bazett) 325
QTC (Framingham) 356
QTC (Friderica) 335
QTC (Call) 331

The individual carrying this image was the second patient on our files. He had persistent and but nonspecific symptoms, mainly palpitations and paroxysmal nighttime dyspnoea. He was visiting multiple hospitals and private clinics.

The last electrocardiographic image belongs to a 21-year-old woman, with prodromal palpitations both at rest and while she was walking, on occasion followed by syncope. This had happened to her since childhood (more than ten well-described events in her 21 years); she was treated as if she had epileptic convulsions, despite the fact that no classical abnormality had been seen in her electroencephalographic studies. Later, she was considered to suffer from "cardiac neurosis". In fact, she carried the pattern of "short PR and QTc interval".

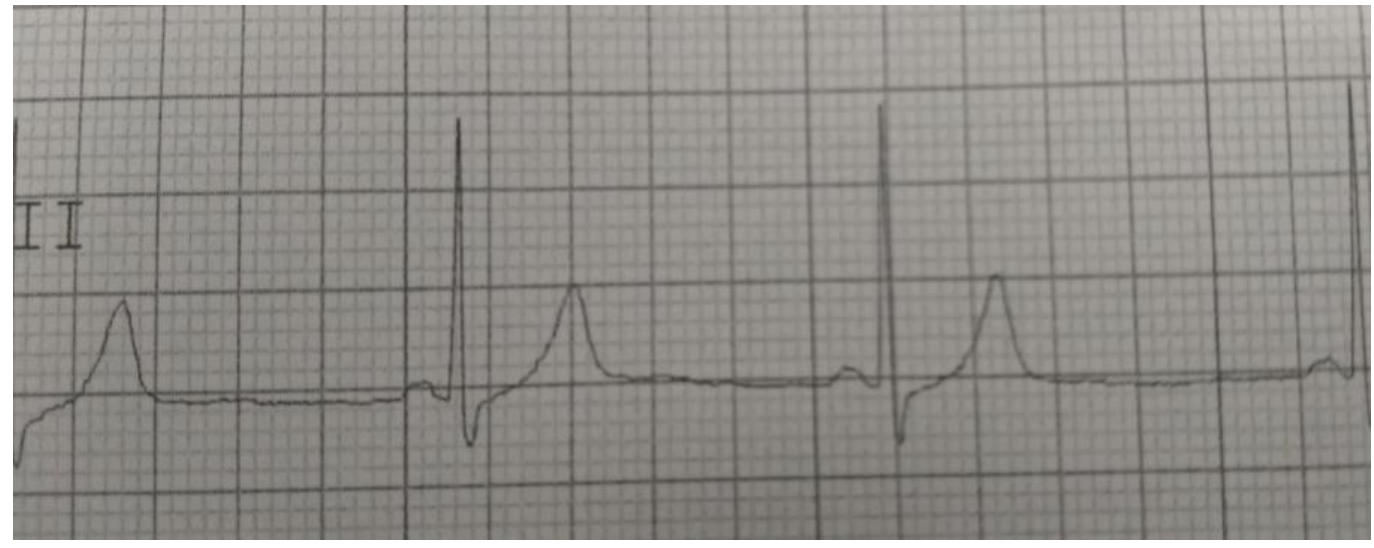

Figure 6. Enlarged image on D2 derivation and corresponding corrected QT interval values.

*Average value in PR interval length: 108-112 milliseconds.

*Average heart rate measured: 55-58 bpm.

Corrected QT length measurements: $<370$ milliseconds.

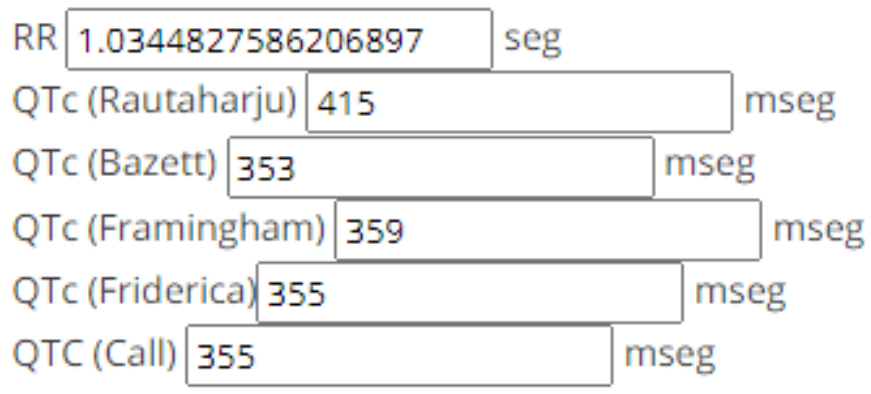


The main symptoms reported by patients carrying the entity described over a universe of 330 patients with a diagnosis of certainty for such.

Table 1. Graphics (detailed)
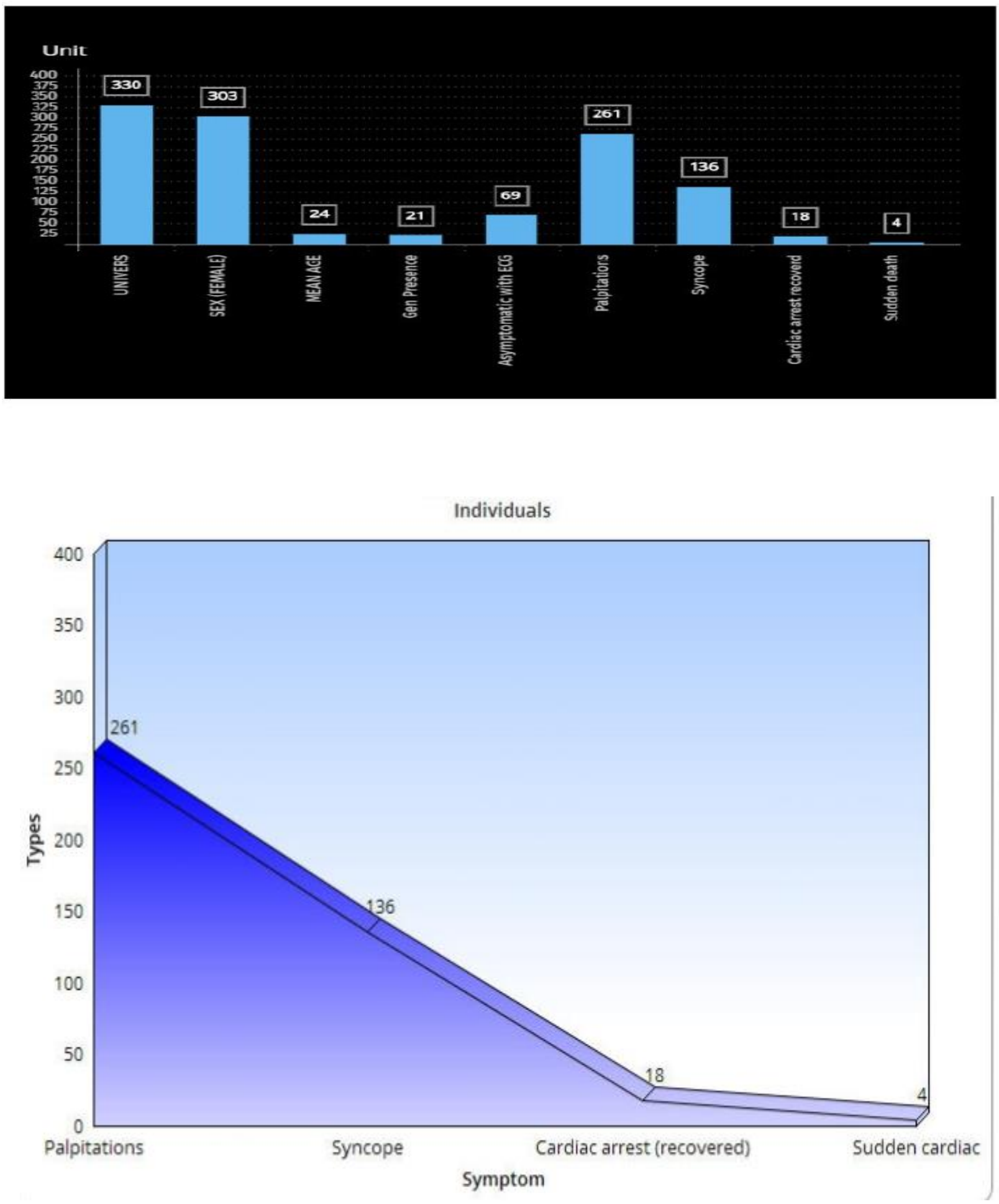
These integer values reflected in the graphs can also be seen and assessed in the following table.

Both in their integer values (number of patients who are presenting some symptom and as a percentage thereof out of 330 patients evaluated in this clinical study.

Table 2. Overall numbers and Percentages

\begin{tabular}{ccc}
\hline $\begin{array}{c}330 \\
\text { TOTAL PATIENTS }\end{array}$ & INTEGER VALUES & IN PERCENTAGE \\
\hline Male sex/Female sex & $27 / 303$ & $8.18 / 91.81$ \\
\hline Average age & 24 years & 6.36 \\
\hline Presence of gene & 21 & 20.90 \\
\hline $\begin{array}{c}\text { No symptoms with } \\
\text { Positive ECG }\end{array}$ & 69 & 79.09 \\
\hline Symptomatic & 261 & 93.33 \\
\hline *Slight symptoms & 308 & 6.66 \\
\hline$*$ Very severe symptoms & 22 & \\
\hline
\end{tabular}

* Slight symptoms are mainly:

Compulsive health observation. (They visit many doctors in search of different views).

Nervousness, Apathy, Crying, Insomnia or drowsiness. Palpitations at night rest.

** Severe and very severe symptoms:

Intense precordial palpitations at night rest and awakening the patient from physiological sleep (261). Recurrent syncope attacks (136). Recovered cardiac arrest (18). Sudden cardiac death (4).

Although some affected patients may never report any symptoms, being detected only by meticulous electrocardiographic study, the most common symptoms (Table 2) of our patients, as previously described, are multiple and overlapping. In order of frequency, the most prevalent symptom is that of "palpitations that wake up the patient from sleep". Commonly affected patients may visit emergency departments for troublesome palpitations. In our series palpitations (described as irregularities of the heartbeat characterized by an awareness of them on the anterior region of the chest, and by hard, fast and/or irregular heartbeats) are the number one symptom. Also frequent are complaints of repetitive loss of consciousness, of short duration, without loss of sphincter control and usually with complete recovery (in many cases confirmed by direct witnesses) This syncope is the number two symptom in this kind of patient. Frequently patients report feeling the palpitations immediately before losing consciousness. Lightheartedness (sometimes inappropriately called presyncope) is common but may not be of clinical importance.

Although sudden death is not frequent in this ECG pattern, it can appear. Palpitations without apparent cause, reported syncope, and the fact that the simultaneously short PR and QT intervals may go unnoticed, are the main reasons why such patients may be referred to the psychiatric services, being frequently diagnosed with "cardiac neurosis

As would be easy to understand, these symptoms do not necessarily appear in isolation.

Most of the time they appear "imbricated", hence, and as a very common example, the first symptom that the individual feels is the sensation of very fast and strong palpitations (hitting the chest), and then syncope may or may not appears.

In other cases, the symptoms are very unspecific, always mild but all present the typical electrocardiographic pattern. 


\section{Discussion}

We propose the electrocardiographic pattern of shortening of the PR and QTc intervals in the same tracing and fulfilling the criteria of a PR interval less than 120 milliseconds and the QTc interval less than 350 milliseconds (depending on gender) is a distinct syndrome, which we believe can seriously threaten the life of the person suffering from it.

Alterations of the electrocardiographic intervals are well known. Both a short PR interval (or PQ interval for some authors) and a short QT interval (corrected by various calculation formulas) are being much more frequently diagnosed compared to only a decade ago. A short PR interval ( $<120 \mathrm{msec}$.) for example can be associated with a variety of symptoms such as palpitations and syncope. Clinically, the individual may be asymptomatic or experience a variety of complex arrhythmias, which can be disabling but very seldom result in sudden death.

A short QT (equal to less than $360 \mathrm{msec}$ in men and equal or less than $370 \mathrm{msec}$. in women) refers to the electrocardiographic manifestation of an accelerated cardiac repolarization. Acquired disease - the most common cause - can result from electrolyte disturbances or drugs, as well as respiratory or metabolic acidosis. It can manifest with atrial and ventricular arrhythmias, and even sudden cardiac death. Cardiac arrest occurs as the presenting symptom in up to $3-4 \%$ of the cases, but fortunately, many can be resuscitated. Simultaneous shortening of the PR and QT intervals is the electorcardiographic manifestation of an accelerated cardiac repolarization. The electrocardiographic pattern of short $\mathrm{PR}$ and QT intervals together in the same electrocardiographic tracing (Breijo pattern) [2] was described more recently (2008), but that does not make it any less important for an accurate diagnosis and clinical management.

The most well-known entities for causing a short PR interval are the following:

- $\quad$ *Wolff-Parkinson-White (W-P-W).[4]

- $\quad *$ Breijo's Pattern [2].

- $\quad{ }^{*}$ Lown-Ganonl- Levine (L-G-L).[5]

- $\quad$ * Mannheim Pattern.[6]

All of them have a short PR interval, but the differential diagnosis must be taken into account, since they are not the same cardiological entity (Table 3).

Table 3. Differential diagnosis between entities with a short PR interval (Less than 120 milliseconds).

\begin{tabular}{|cccc|}
\hline ENTITY & PR interval length & QRS complex length & $\begin{array}{c}\text { Corrected QT interval } \\
\text { length }\end{array}$ \\
W.P.W & Short (delta wave) & Wide & Normal \\
L-G-L & Short & Normal & Normal \\
Mannheim & Short or normal & Wide or normal & Normal \\
Breijo Pattern & Short or very short & Normal & Short or very Short \\
\hline
\end{tabular}

They all share - in having a short PR - that the cells of the heart are more unstable and more vulnerable to any stimulus - either endogenous or exogenous - which could predispose to serious arrhythmia (ventricular fibrillation, cardiac arrest or sudden cardiac death) if the provoking stimuli are strong enough. Fortunately, the such events remain relatively rare; Gollop et al. [3] having described about only 61 cases of short QT interval.

Their cohort of 61 cases was predominantly male $(75.4 \%)$ and had a mean QTc value of $306 \mathrm{~ms}$ with values ranging from 248 to $381 \mathrm{~ms}$ in symptomatic cases. In Gallop et al's report the overall median age at clinical presentation was 21 years [IQR: 17-31.8 years] 
with a value of 20 years (IQR: 17-29 years) in men and 30 years (IQR: 19 -44 years) in women. These authors also described the characteristics of the ECG in the general population and, taking into account the clinical presentation, family history and genetic findings, suggested a diagnosis of high sensitivity could be made through their proposed scoring system.

This risk score can be seen below.

\begin{tabular}{|c|c|}
\hline QT interval value (in msec) & \\
\hline$<370$ milliseconds & 1 points \\
\hline$<350$ milliseconds & 2 points \\
\hline$<330$ milliseconds & 3 points \\
\hline $\mathrm{J}$ point-T peak interval & 1 point \\
\hline \multicolumn{2}{|l|}{ Clinical history } \\
\hline Sudden cardiac arrest & 2 points \\
\hline Polymorphic VT or VF & 2 points \\
\hline Unexplained syncope & 1 points \\
\hline Atrial fibrillation & 1 points \\
\hline \multicolumn{2}{|l|}{ Family history } \\
\hline First or second degree relative to SQTS & 2 points \\
\hline First or second degree relative to sudden death & 1 point \\
\hline Sudden infant death syndrome & 1 point \\
\hline \multicolumn{2}{|l|}{ Genotype } \\
\hline Genotype positive (KCNE3 for us) & 2 points \\
\hline Mutation of undetermined significance in a culprit gene & 1 points \\
\hline
\end{tabular}

Patients can be sub-classified into high probability ( $\geq 4$ points), intermediate probability ( 3 points) or low probability ( $\leq 2$ points). These values coincide with ours. In the cases evaluated by us, women have been more prevalent than men by far. The average age of the patients has been similar.

Gaita et al. [6] published in 2003 an excellent article in "Circulation" entitled: "Short QT syndrome. A familiar cause of sudden death", as have other reports such as Bjerregaard's in 2018 [8].

Since the first description of the short QT interval there have been many publications about it. However, the association of both a short PR interval and a short QTc in the same person, and in the same electrocardiographic tracing was first described by Breijo et al [2]. in 2008, published in the International Journal of Cardiology with the title: "Decrease in cardiac electrical systole". This so-called "Breijo pattern" can also lead to serious events - cardiac arrest due to ventricular fibrillation - and even sudden death, although the most frequent symptoms in our cases, are more annoying than life-threatening. Thus although the clinical-electrocardiographic condition that we describe can a threat to life, that remains unusual.

The so-called "short QT syndrome" has also been described by many other authors.

Authors such as Gussack, Gaita [7] and many others [8] described it as the cause of sudden deaths, with hereditary characteristics. But we have always been struck by the great discrepancy between the authors themselves in setting the limits on the length of the QT interval to be be considered as short. Gollop et al [3], have unified criteria in this 
respect and we also consider as short a QT interval when it is less than 370 milliseconds in females and 360 milliseconds in males.

QT and PR shortening have always been diagnosed separately, that is: short PR interval and short QT interval as isolated entities. But until our first description, never had before both entities had been described in the same ECG tracing. In this paper, we describe a frequent pattern and describe its clinical-electrocardiographic features.

More than 330 patients have been registered in our files (with $6.36 \%$ of the patients carrying an altered KCNE3 gene) so we could deduce that the entity has possibly two forms, one being genetic. If it were only one or two cases, we could think that these are only isolated and anecdotal cases. But with 330 patients carrying such an entity, we can contend it is real and not uncommon finding. Many authors have considered "cardiac electrical systole" as the period that begins from the ascending branch of the P wave and lasts to the descending branch of the T wave, until it reaches the ECG isoelectric line tracing.

In other words, depolarization of both atria and ventricles, and not just ventricular depolarization. The shortening of cardiac electrical systole is nothing more than the "presence of a short PR interval and a short QTc interval" in the same electrocardiographic tracing, accompanied by a number of quite typical - though by no means pathognomonic - symptoms. This genetic condition, mediated by the above-mentioned gene (KCNE3 in its ectopic expression [1]) and transmitted in a recessive way, makes the cardiac electrical system very much vulnerable and susceptible to produce very harmful results in the person who suffers from it. However, we also have to say there are many patients with the same electrocardiographic pattern (short PR and QT intervals) and similar symptoms, in whom the above-mentioned gene has not been detected in any of its variants. Only 6$36 \%$ of the individuals in our series with the electrocardiographic pattern of short PQ and QTc intervals had the aforementioned gene present. Therefore not all the carriers of the typical electrocardiographic tracing have the abnormal gene and not all the carriers of the typical electrocardiographic tracing have symptoms. The electrocardiographic entity we describe of short PR and QT intervals. is frequently passed off as "normal".

We suggest that symptoms such as palpitations syncope and others correlate should lead to detailed evaluation of the ECG looking for "intermittent" short PR intervals and short QT intervals. In the case of a pattern such as the one described, with short PR and QT intervals in the same trace, when the patient is in "baseline conditions", the electrocardiographic tracing appears at first sight to be normal, with a tendency to a low heart rate - although never less than 50 beats per minute.

The symptoms referred by patients with this electrocardiographic anomaly, fortunately, in most cases do not threaten life. They tend to be mild, although tremendously annoying for the sufferer, so much so that these individuals can be habitual visitors to the emergency services, from where they may be transferred to psychiatric services in the belief that it is a case of "cardiac neurosis". On the other hand a considerable number of subjects who have this electrocardiographic anomaly may not present with symptoms during their lifetime. And when they die from any other pathology, the necropsies may show a structurally normal heart. The symptoms described by patients with this electrocardiographic pattern range from total asymptomatology, to the sensation of imminent fall, nocturnal palpitations that awaken individuals from sleep, a total loss of consciousness (syncope) usually of short duration according to witnesses who have been able to see it - and with total recovery after the syncope. On many occasions, all these symptoms appear overlapped. The great annoyance and anxiety of the patients is that such symptoms are usually repetitive and without a diagnosis that justifies them. 


\section{Conclusions}

We describe a common ECG pattern, associated with troublesome symptoms, which frequently goes unnoticed and is often considered as normal. Of series of 330 very well evaluated patients, all fulfill two essential and necessary requirements:

1. All they have a short PR interval, lower than 120 milliseconds.

2. All they also have a short corrected QT interval: Lower than 370 milliseconds in women and lower than 360 milliseconds in men.

Although a considerable percentage of these patients are asymptomatic throughout their lives and are only recognized by routine ECG studies, another large percentage do have symptoms, often from childhood. When they are symptomatic, the most frequent symptoms are:

1. Palpitations, normally nocturnal and " announcing " an imminent syncope.

2. True syncope which may be confused with epileptic seizures

Sudden cardiac arrest is fortunately rare. Only in 6-36\% (21 people out of 330 tested) of the individuals screened and assayed as carriers of the electrocardiographic pattern of short PQ and QTc intervals was a gene abnormality detected. In our research on this subject, women were much more frequent to suffer from the entity than men. The overall average age of the 330 individuals evaluated and confirmed as carriers of this entity was 23.9 old years.

\section{Declaration of interests}

Both the author and his closest collaborators in this clinical work declare that they have no conflict of interest.

\section{Acknowledgments}

The authors state that they abide by the Requirements for Ethical Publishing in Biomedical Journals. [11].

Sincere thanks to Drs:

Johnson Jayden PhD.

O'DonNell. Alexander PhD.

Pardo Rios Manuel. PhD.

They made possible the electrocardiographic "blinded" study in order to avoid interpretation biases.

My sincere thanks also go to the Cardiology Departments of Hospital Angeles Mexico D.C. Saint Francis Hospital (Hartford, Connecticut), East Boston Hospital (Boston, Massachusetts), as well as the primary care centers in Hartford (CT), Boston (MA) and Albacete (Spain).

Many thanks to all of them.

\section{References}

[1] Reza Mazhari, H. Bradley Nuss et al. Ectopic expression of KCNE3 accelerates cardiac repolarization and abbreviates the QT interval. J Clin Invest. 2002; 15; 109(8): 1083-1090. https://doi.org/10.1172/jci15062

[2] Francisco R. Breijo-Marquez. Decrease of electrical cardiac systole. 126, 2, E36-E38, MAY 23,2008. https://doi.org/10.1016/j.ijcard.2007.12.051

[3] Gollob MH, Redpath CJ, Roberts JD et al. The Short QT syndrome Proposed Diagnostic Criteria. J Am Coll Cardiol. 2011 ; 57. https://doi.org/10.1016/j.jacc.2010.09.048

[4] L. Wolff, J. Parkinson, P. D. White. Bundle-branch block with short P-R interval in healthy young people prone to paroxysmal tachycardia. American Heart Journal, St. Louis, 1930, 5: 685. https://doi.org/10.1111/j.1542-474x.2006.00127.x

[5] Lown B, Ganong WF, Levine SA. The syndrome of short P-R interval, normal QRS complex and paroxysmal rapid heart action. Circulation. 1952; (5): 693-706.https://doi.org/10.1161/01.cir.5.5.693

[6] Mahaim J, Benatt A. Et al. Nouvelles recherches sur les connections superieures de la branche du faisceau de His-Tawara avec cloison interventriculaire. Cardiologia. 1937; 1: 61-8. https://doi.org/10.1159/000164567 
[7] Fiorenzo Gaita. Carla Giustetto. et al. Short QT Syndrome A Familial Cause of Sudden Death. Circulation. 2003;108:965-970. https://doi.org/10.1161/01.CIR.0000085071.28695.C4

[8] Bjerregaard P . Diagnosis and management of short QT syndrome. Heart Rhythm. 2018 15 (8): DOI: https://doi.org/10.1016/j.hrthm.2018.02.034

[9] Breijo-Marquez FR . A Breijo Pattern Associated to a Wolff-Parkinson-White Pattern. J Cardiol Curr Res. 2016; 5(3): 00161.https://doi.org/10.15406/jccr.2016.05.00161

[10] Francisco R. Breijo-Márquez. Breijo Electrocardiographic Pattern, Cardiac Arrhythmias. 2018; Umashankar Lakshmanadoss, IntechOpen, DOI: 10.5772/intechopen.75446. Available from: https://www.intechopen.com/books/cardiac-arrhythmias/breijoelectrocardiographic-pattern. https://DOI: 10.5772/intechopen.75446.

[11] Shewan LG, Coats AJS, Henein MY.' Responsibilities and Ethical Publishing. International Cardiovascular Forum Journal 2018;13:3-4. DOI:10.17987/icfj.v13i0.525. 